\title{
Evaluating the Microbiome of Hemp
}

\author{
Samuel E. Barnett, ${ }^{1}$ Ali R. Cala, ${ }^{2}$ Julie L. Hansen, ${ }^{3}$ Jamie Crawford, ${ }^{3}$ Donald R. Viands, ${ }^{3}$ Lawrence B. Smart, ${ }^{4}$ Christine D. Smart, ${ }^{2, \dagger}$ \\ and Daniel H. Buckley ${ }^{1}$ \\ ${ }^{1}$ Soil and Crop Sciences Section, School of Integrative Plant Science, Cornell University, Ithaca, NY 14853, U.S.A. \\ ${ }^{2}$ Plant Pathology and Plant-Microbe Biology Section, School of Integrative Plant Science, Cornell University, Cornell AgriTech, Geneva, NY \\ 14456, U.S.A. \\ ${ }^{3}$ Plant Breeding and Genetics Section, School of Integrative Plant Science, Cornell University, Ithaca, NY 14853, U.S.A. \\ ${ }^{4}$ Horticulture Section, School of Integrative Plant Science, Cornell University, Cornell AgriTech, Geneva, NY 14456, U.S.A.
}

Accepted for publication 29 August 2020.

\section{ABSTRACT}

Plant microbiomes contribute to plant fitness and crop yields through a variety of mechanisms. Determining variability in microbiome composition among individuals of a species, and identifying core microbiome membership, are essential first steps for exploring host-microbe interactions. Members of a core microbiome are microorganisms that are tightly associated with and are found widespread across individuals of a plant genotype or species. Hemp (Cannabis sativa L.) is an economically important crop that has gained a resurgence following its removal from the list of controlled substances by the U.S. government. Despite renewed interest in this crop, the microbiome of hemp has not been well studied. We analyzed the bacterial and fungal communities associated with four plant compartments (rhizosphere, root tissue, leaf surface, and flowers) of $C$. sativa 'Anka' across six fields in the Finger Lakes region of New York, United States. We found that both bacterial and fungal community composition varied significantly among plant compartments. Rhizosphere communities were largely similar to the bulk soil communities but root tissue, leaf, and flower communities had distinct compositions. We identified candidate core microbiome members of each plant compartment (bacterial core taxa: root tissue $[n=6]$, leaves [ $n=$ $11]$, and flowers [ $n=7]$; fungal core taxa: rhizosphere $[n=1]$, leaves [ $n=14]$, and flowers [ $n=2]$ ). Many of these candidate core microbiome members were related to organisms previously associated with plant growth promotion or pathogen resistance in various plants. The core microbiome identified in this study can be further investigated to improve cultivation of this important crop.

Keywords: bacteria, Cannabis sativa, crop, fungi, hemp, microbiome, microorganism, phyllosphere, rhizosphere
Plant-associated microbes are key players in the health and productivity of their plant hosts (Turner et al. 2013). Plant microbiomes include bacteria and fungi within rhizosphere soil, within root tissues (root epiphytes, ectophytes, and endophytes), and upon aboveground structures such as leaves and flowers

\section{Corresponding author: C. D. Smart; cds14@cornell.edu}

Author contributions: C.D.S. and A.R.C. designed the experiment with consultation from D.H.B. A.R.C., J.L.H., J.C., D.R.V., L.B.S., and C.D.S. designed and implemented hemp cultivation and sample collection. A.R.C. and S.E.B. performed the experiments and collected the results. S.E.B. analyzed the data and wrote the manuscript with much input from A.R.C., C.D.S., and D.H.B.. All authors contributed to editing the manuscript.

Funding: Support was provided by the New York State Department of Agriculture and Markets (grant number C00195GG).

The author(s) declare no conflict of interest. (phyllosphere) (Turner et al. 2013). Some of these microbes perform beneficial functions for their hosts, including fixation of atmospheric nitrogen (Franche et al. 2009), improving access to soil nutrients (Colombo et al. 2014; Gyaneshwar et al. 2002; Whiting et al. 2001), production of plant hormones (Boller 1995; OrtízCastro et al. 2009), protecting against pathogens through competitive exclusion (Innerebner et al. 2011), production of antimicrobial compounds (Lievens et al. 1989; Urquhart and Punja 2002; Weller 1988), and plant defense priming (Pieterse et al. 2014). The microbiome's role in plant health has made it a target for study in economically important crops such as maize, soybean, and rice (de Cássia Silva et al. 2016; Edwards et al. 2015; Kuklinsky-Sobral et al. 2004; Peiffer et al. 2013; Wallace et al. 2018).

High-throughput DNA sequencing has promoted numerous studies describing the microbiomes of important agricultural plant species (Busby et al. 2017). These early reports are important stepping stones for understanding how microbiomes affect plant growth, identifying the impact of plant traits on microbiome composition, and developing methods for manipulating microbiomes to increase crop yields or disease resistance (Busby et al. 
2017). Microbiome considerations are especially important when developing sustainable cropping systems (Toju et al. 2018).

Hemp is likely to be an important crop in the United States, with economic value in many industries such as textiles and fibers, food products, personal care products, and health and wellness products (Cherney and Small 2016). Hemp refers to cultivars of Cannabis sativa with less than $0.3 \%$ dry weight tetrahydrocannabinol (THC) (Small and Cronquist 1976). Recent U.S. government legislation has removed hemp from the list of controlled substances, reducing roadblocks to cultivation and promoting agricultural development for this crop (115th Congress 2018). Despite the anticipated growth of hemp cultivation, little is known about its microbiome. Almost all previous studies examining the microbiome or generally microbes associated with $C$. sativa focus on high-THC cultivars grown primarily for recreational or medicinal use (Comeau et al. 2020; McKernan et al. 2015; Winston et al. 2014). Others focused on examining activities of isolated $C$. sativa endophytes (Gautam et al. 2013; Kusari et al. 2013; Scott et al. 2018).

Here, we present one of the first characterizations of both bacterial and fungal communities associated with multiple plant compartments of hemp. We used high-throughput sequencing of bacterial (16S ribosomal RNA [rRNA] gene) and fungal (internal transcribed spacer 1 [ITS1]) biomarkers to describe the microbiome of the $C$. sativa 'Anka' grown across six field sites in the Finger Lakes region of New York, United States. We examined the microbes inhabiting the rhizosphere, root tissue, leaf surface, and flower surfaces, aiming to identify the core microbiome of Anka grown in this region. Results from our study will provide a reference for future research in defining functionality of microbial symbionts of hemp and developing sustainable microbiome manipulations to improve crop yields.

\section{MATERIALS AND METHODS}

Plant cultivation and sampling. Plants used in this study were part of a larger cultivar trial (35 entries) and were planted in six secure research field locations around the Finger Lakes region of New York (Table 1$)$. Plot trials $(1.22$ by $6.10 \mathrm{~m}$ ) were planted with a tractor-mounted 6-row cone seeder. In McGowan Early, McGowan Late, East Ithaca, and Research North fields, nitrogen was applied at7.85 $\mathrm{g} \mathrm{m}^{-2} 3$ weeks after planting. At Crittenden North, nitrogen at $11.21 \mathrm{~g} \mathrm{~m}^{-2}$ was applied prior to planting. No nitrogen was applied at Freeville. Each plot was replicated five times in a randomized complete block design. All microbiome sampling was done on C. sativa 'Anka' (Uniseeds, Ontario, CA, U.S.A.), which is a monecious cultivar used for dual-purpose grain and fiber production. The seeding rate was pure live seed at $2.24 \mathrm{~g} \mathrm{~m}^{-2}$.

Five plants were collected per field (one plant per replicate plot) when plants were in full flower (Table 1) and most plants were over $2 \mathrm{~m}$ in height. For each collection, the entire plant was dug up and placed in a large plastic bag. Bulk soil was collected to a depth of $10 \mathrm{~cm}$ within $1 \mathrm{~m}$ of the selected plant and placed in a Ziplock bag. Samples were driven to the laboratory for immediate processing or placed in a cold room $\left(4^{\circ} \mathrm{C}\right)$ and processed within $24 \mathrm{~h}$. To process plants, large soil aggregates were first removed from the roots; then, the rhizosphere soil was gently removed from roots and collected. The roots were cut into 4-cm-long pieces using a razor blade. Rhizosphere and bulk soil samples were sieved to $2 \mathrm{~mm}$ and stored at $-20^{\circ} \mathrm{C}$ prior to DNA extraction. Roots were frozen with liquid nitrogen and crushed using a mortar and pestle, then stored at $-20^{\circ} \mathrm{C}$ prior to DNA extraction.

For leaf and flower samples, we specifically targeted collection of bacterial and fungal epiphytes. Cotton swabs were dipped in sampling buffer (100 mM potassium phosphate, $\mathrm{pH} \mathrm{7;10} \mathrm{mM}$ EDTA; and $0.05 \%$ Triton), then rubbed over either the leaf or flower surface for $10 \mathrm{~s}$ The swab was then returned to a tube with $1 \mathrm{ml}$ of sampling buffer. To concentrate the flower and leaf wash samples for DNA extraction, they were lyophilized at $-40^{\circ} \mathrm{C}$, then rehydrated in $200 \mu \mathrm{l}$ of molecular-grade water. Samples were stored at $-20^{\circ} \mathrm{C}$ prior to DNA extraction.

DNA extraction, amplification, and sequencing. We extracted DNA from samples with the PowerMag Microbiome RNA/DNA Isolation Kit (catalog number 27500-4-EP; MO BIO Laboratories Inc., Carlsbad, CA, U.S.A.), using the manufacturer's instructions with the following modifications. We used $0.25 \mathrm{~g}$ of bulk or rhizosphere soil per soil DNA extraction. For root tissue extraction, between 0.10 and $0.25 \mathrm{~g}$ of powdered root tissue was used due to limited material. All $200 \mu$ l of rehydrated wash solution was used for extraction from leaf and flower washes. All samples underwent bead beating for $2.5 \mathrm{~min}$ using a Mini-Beadbeater-96 (catalog number 1001; Biospec Products, Bartlesville, OK, U.S.A.).

Prior to amplification, we quantified DNA concentrations using a Quant-iT PicoGreen assay (number P7589; Thermo Fisher Scientific, Waltham, MA, U.S.A.). High-concentration samples were

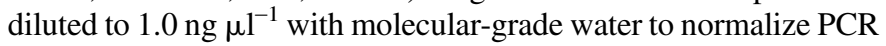
template concentrations. To capture the bacterial community composition, we amplified the V4 region of the 16S rRNA gene using the 515f/806r dual-indexing primer set (Kozich et al. 2013). To capture the fungal community composition, we amplified the ITS1 region using the nBITS2f/58A2r primer set (Koechli et al. 2019). The nBITS2f/58A2r primer set used the same dual-indexing scheme as the $515 \mathrm{f} / 806 \mathrm{r}$ primer set. Triplicate PCR assays contained $2.0 \mu \mathrm{l}$ of template, $2.5 \mu \mathrm{l}$ of pooled $10 \times$ forward and reverse primers, $13.1 \mu \mathrm{l}$ of Q5 master mix (catalog number M0494L; New England Biolabs, Ipswich, MA, U.S.A.) containing 1:0.025 (vol/ vol) $4 \times$ PicoGreen reagent, and $7.4 \mu \mathrm{l}$ of molecular-grade water. PCR conditions were $95^{\circ} \mathrm{C}$ for $2 \mathrm{~min}$; followed by 30 cycles of $95^{\circ} \mathrm{C}$ for $20 \mathrm{~s}, 55^{\circ} \mathrm{C}$ for $15 \mathrm{~s}$, and $72^{\circ} \mathrm{C}$ for $10 \mathrm{~s}$; and followed by $72^{\circ} \mathrm{C}$ for $5 \mathrm{~min}$. We normalized PCR product concentrations across samples, replicates pooled, with the Invitrogen SequalPrep Normalization Plate Kit (catalog number A1051001; Thermo Fisher Scientific, Waltham, MA, U.S.A.), then pooled 16S rRNA gene and ITS1

TABLE 1

Description of field sites, hemp planting dates, sample collection dates, and grain yield

\begin{tabular}{llllrr}
\hline Site name & Location & \multicolumn{1}{c}{ Soil type } & Planting date & Collection date & Grain yield \\
\hline East Ithaca & Ithaca, NY & Arkport fine sandy loam & 14 June 2017 & 2 August 2017 & 582.20 \\
\hline McGowan & Ithaca, NY & Niagara silt loam & 9 June 2017 & 26 July 2017 & $2,340.80$ \\
\hline McGowan Late & Ithaca, NY & Niagara silt loam & 29 June 2017 & 9 August 2017 & $1,608.60$ \\
\hline Research North & Geneva, NY & Lima loam & 28 June 2017 & 10 August 2017 & 624.22 \\
\hline Crittenden North & Geneva, NY & Odessa silt loam & 10 July 2017 & 21 August 2017 & 780.27 \\
\hline Freeville & Freeville, NY & Howard gravelly loam & 12 July 2017 & 23 August 2017 & NA \\
\hline
\end{tabular}


libraries separately. Libraries were sequenced at the Cornell Biotechnology Resource Center (Ithaca, NY, U.S.A.) on the Illumina MiSeq platform with the paired-end 2-by-250-bp V2 kit for the $16 \mathrm{~S}$ rRNA gene library and the paired-end 2-by-300-bp V3 kit for ITS1 library. Raw demultiplexed reads are accessible on the short-read archive with BioProject accession number PRJNA607742.

Sequence processing. For both amplicon libraries, we merged paired-end reads using PEAR (Zhang et al. 2014) with default settings, then demultiplexed using a custom script. For the $16 \mathrm{~S}$ rRNA gene amplicon library, we performed alignment-based quality filtering with mothur (Schloss et al. 2009) with the Silva SEED database release 128 as a reference, discarding homopolymers greater than $8 \mathrm{bp}$. Reads mapping to mitochondria and chloroplasts were removed. We clustered operational taxonomic units (OTUs) at $97 \%$ sequence identity with UPARSE clustering from USEARCH (Edgar 2010). Taxonomy was assigned using the uclust algorithm through QIIME (Caporaso et al. 2010) with the SILVA release 128 database as reference. OTUs not classified as bacteria were removed.

For the ITS1 amplicon library, we used mothur (Schloss et al. 2009) for quality filtering, with a maximum homopolymer length of 8 bp but without alignment-based filtering. We then filtered and trimmed sequences to just the ITS1 region using ITSx (BengtssonPalme et al. 2013). To improve OTU clustering, all sequences were set to an equal length by adding ambiguous bases $(\mathrm{N})$ to the ends of each sequence (Palmer et al. 2018). Using vsearch (Rognes et al. 2016), we preclustered sequences at $98 \%$ sequence identity and filtered out chimeric sequences. We then clustered final OTUs at $97 \%$ sequence identity. Taxonomy was assigned using the sintax classifier through vsearch with the USEARCH formatted UNITE reference database, release 01.12.2017. OTUs not classified as fungi were removed. The sequence processing pipeline, representative OTU sequences, OTU and taxonomy tables, and metadata are available at https://github.com/seb369/hemp_microbiome (archived via Zenodo) (Barnett 2020).

Data analysis. We performed all data analysis in $\mathrm{R}$ version 3.6.2 ( $\mathrm{R}$ Core Team 2018) and performed each analysis separately for fungi and bacteria. Code for all analyses are available at https:// github.com/seb369/hemp_microbiome. All root tissue samples were removed from the fungal amplicon library because the vast majority of reads matched the $C$. sativa ITS1 region, leaving too few actual fungal reads for analysis. We further removed samples with less than 2,000 bacterial reads or 1,000 fungal reads prior to analysis because these cutoffs typically indicated poorly sequenced samples. This read cutoff removed some replicate plants; thus, although 30 plants were originally sampled, often less than 30 plants were analyzed. Read counts varied across samples, from 2,425 to 223,698 bacterial reads and 1,396 to 58,300 fungal reads. In order to account for different sequencing depths between samples, we rarefied read counts to that of the lowest read count sample (bacterial $=2,425$ and fungal $=1,396$ ) using the rarefy_even_depth function from package phyloseq (McMurdie and Holmes 2013). Rarefaction was used to normalize sequence counts because it is among the most robust methods for normalizing uneven sample sizes (Weiss et al. 2017). One drawback to this normalization method is that it results in random loss of some OTUs within samples despite their detection originally. This may disproportionately lead to false-negative detection of low-abundance OTUs or taxa susceptible to primer bias. Species evenness was calculated for each sample and the effect of plant compartment on evenness was determined by analysis of variance (ANOVA). The post hoc Tukey test of evenness was run between plant compartments using function glht from package multcomp (Hothorn et al. 2008).
To compare overall bacterial or fungal community structure across plant compartment and field locations, we ran a permutational multivariate ANOVA (PERMANOVA) on the Bray-Curtis dissimilarity metric with the function adonis from package vegan (Oksanen et al. 2018). The PERMANOVA models incorporated plant compartment, field, and their interaction as variables. We generated ordinations based on the Bray-Curtis dissimilarity metric using function ordination from package phyloseq (McMurdie and Holmes 2013). To examine the interaction between plant compartment and field site on community composition, we ran separate PERMANOVA on Bray-Curtis dissimilarity within each plant compartment with field as the sole variable, with $P$ values corrected for multiple comparisons using Bonferroni correction. This analysis was to determine whether field site was an important factor in community composition for each plant compartment.

Although we were interested in the effect of the microbial community compositions on crop productivity, we were limited in our data on crop yield, because this value was measured for fields and not individual plants. No yield data were collected from the Freeville field. To see whether or not community composition differences corresponded to differences in grain yield, we averaged the Bray-Curtis dissimilarity across all sample comparisons between each field pair and then calculated the Pearson correlation coefficient between these community dissimilarity measures and the difference in grain yield between the field pairs, with $P$ values corrected for multiple comparisons using Bonferroni correction. This analysis was performed separately for each plant compartment.

We examined the variation in relative abundance for highly abundant phyla or classes ( $>1 \%$ of the bacterial or fungal community) across plant compartments using the Kruskal-Wallis rank sum test with $P$ values adjusted for multiple comparisons with Bonferroni correction. We used a nonparametric test in this case due to the nonnormal nature of these data (Weiss et al. 2017). Separate pairwise comparisons across compartments were then run for taxa with significant abundance variation using the Dunn test function from package FSA (Ogle et al. 2020).

To identify members of the hemp microbiome that may be of further interest, we applied three different approaches. First, we identified the most abundant OTUs within each plant compartment by averaging each OTU's relative abundance across all plants. Relative abundance of these OTUs used the rarefied OTU table. Second, we identified ubiquitous OTUs within each plant compartment (i.e., detected on $100 \%$ of plants). In this case, we used unrarefied OTU tables because uneven sampling depth should have little effect on the type 2 error (i.e., false identification of ubiquitous OTUs). We note that it is likely that some low-abundance yet ubiquitous OTUs were not detected in some samples with lower sequencing depth (i.e., type 1 error). Third, we used DESeq2 (Love et al. 2014) to estimate the $\log _{2}$-fold change enrichment of the OTUs between each plant compartment and the bulk soil. For each DESeq2 analysis, we used unrarefied OTU tables filtered to include only OTUs represented by at least five reads in at least one sample of the plant compartment-bulk soil set. We also used a one-sided test, a $\log _{2}$-fold change threshold of 0.25 , the Wald significance test, the Benjamini and Hochberg $P$ value adjustment for multiple comparisons, and a $P$ value cutoff of 0.1 . We defined the core microbiome as bacterial and fungal OTUs that were ubiquitous in hemp plants and enriched significantly in a plant compartment relative to bulk soil. Presence in the core hemp microbiome does not indicate strength or direction of plant-microbe interaction, nor does it indicate exclusivity to hemp; it merely indicates that the microbes are enriched in hemp across a wide range of soil type and field conditions. 


\section{RESULTS}

Plant compartments harbor different bacterial communities. We identified 8,913 bacterial OTUs across all samples after rarefying, comprising 39 different phyla. We found that plant compartment explained the most variation in microbiome composition (PERMANOVA: $R^{2}=0.32310, \mathrm{df}=4, P=0.001$ ), followed by the interaction of plant compartment and field site $\left(R^{2}=0.16247, \mathrm{df}=\right.$ $20, P=0.001)$, while field site alone explained the least variation in community composition $\left(R^{2}=0.08029, \mathrm{df}=5, P=0.001\right)$. Rhizosphere microbiomes were most similar to those of bulk soil, while root, leaf, and flower microbiomes were all fairly dissimilar in community composition (Fig. 1A). Flower bacterial communities had high variability in community composition, even within a field (Fig. 1A). Field site explained significant variation in bacterial community composition within all plant compartments (bulk soil: $R^{2}=0.4309$, df $=5$, adjusted $P=0.005$; rhizosphere soil: $R^{2}=0.43205$, df $=5$, adjusted $P=0.005$; root tissue: $R^{2}=0.35325$, $\mathrm{df}=5$, adjusted $P=0.005$; leaves: $R^{2}=0.26795, \mathrm{df}=5$, adjusted $P=$ 0.025 ; flowers: $R^{2}=0.32542$, df $=5$, adjusted $P=0.005$ ). However, we found no relationship between bacterial community dissimilarity and crop yield at field scale (rhizosphere soil: Pearson's $r=-0.1056, \mathrm{df}=8$, adjusted $P=1$; root tissue: Pearson's $r=0.2609$, df $=8$, adjusted $P=1$; leaves: Pearson's $r=-0.5691$, df $=8$, adjusted $P=0.3440$; flowers: Pearson's $r=-0.3933$, $\mathrm{df}=8$, adjusted $P=1$ ).

Different bacterial phyla (class for Proteobacteria) were favored in different plant compartments (Fig. 2), with the most abundant taxa ( $>1 \%$ of the bacterial community) varying significantly in relative abundance across plant compartments (Kruskal-Wallis, all
Bonferroni adjusted $P<0.001$ ). Notably, based on post hoc tests (Dunn tests, $P<0.05$ ), no phyla or class varied significantly in relative abundance between bulk and rhizosphere soils. Actinobacteria were in higher relative abundance in the soil, rhizosphere, and root tissue than in either the leaves or flowers. Betaproteobacteria were in greater abundance in root tissue than in any other plant compartment. Alphaproteobacteria were more abundant on leaves than in any other plant compartment. Bacteroidetes were more abundant in root tissue and on leaves than the other plant compartments. Firmicutes and Gammaproteobacteria both had strikingly high abundances on the flowers compared with bulk soil, rhizosphere soil, and leaves, with Gammaproteobacteria also in high abundance in the root tissue (Fig. 2).

Plant compartments harbor different fungal communities. We identified 982 fungal OTUs across all samples after rarefying, comprising 9 phyla. We found that plant compartment explained the most variation in fungal community composition (PERMANOVA: $\left.R^{2}=0.47902, \mathrm{df}=3, P=0.001\right)$, followed by the interaction of plant compartment and field site $\left(R^{2}=0.15231, \mathrm{df}=13, P=0.001\right)$, while field site alone explained the least variation in community composition $\left(R^{2}=0.14361\right.$, df $\left.=5, P=0.001\right)$. Rhizosphere fungal communities were most similar to bulk soil communities, while the fungal communities found on leaves resembled those found on flowers (Fig. 1B). Field site explained significant variation in fungal community composition within all plant compartments (bulk soil: $R^{2}=0.60938$, df $=4$, adjusted $P=0.004$; rhizosphere soil: $R^{2}=$ 0.60956, df $=5$, adjusted $P=0.004$; leaves: $R^{2}=0.4716$, df $=5$, adjusted $P=0.004$; flowers: $R^{2}=0.52618$, df $=5$, adjusted $P=$ $0.004)$. We found no relationship between fungal community dissimilarity and crop yield at field scale (rhizosphere soil:
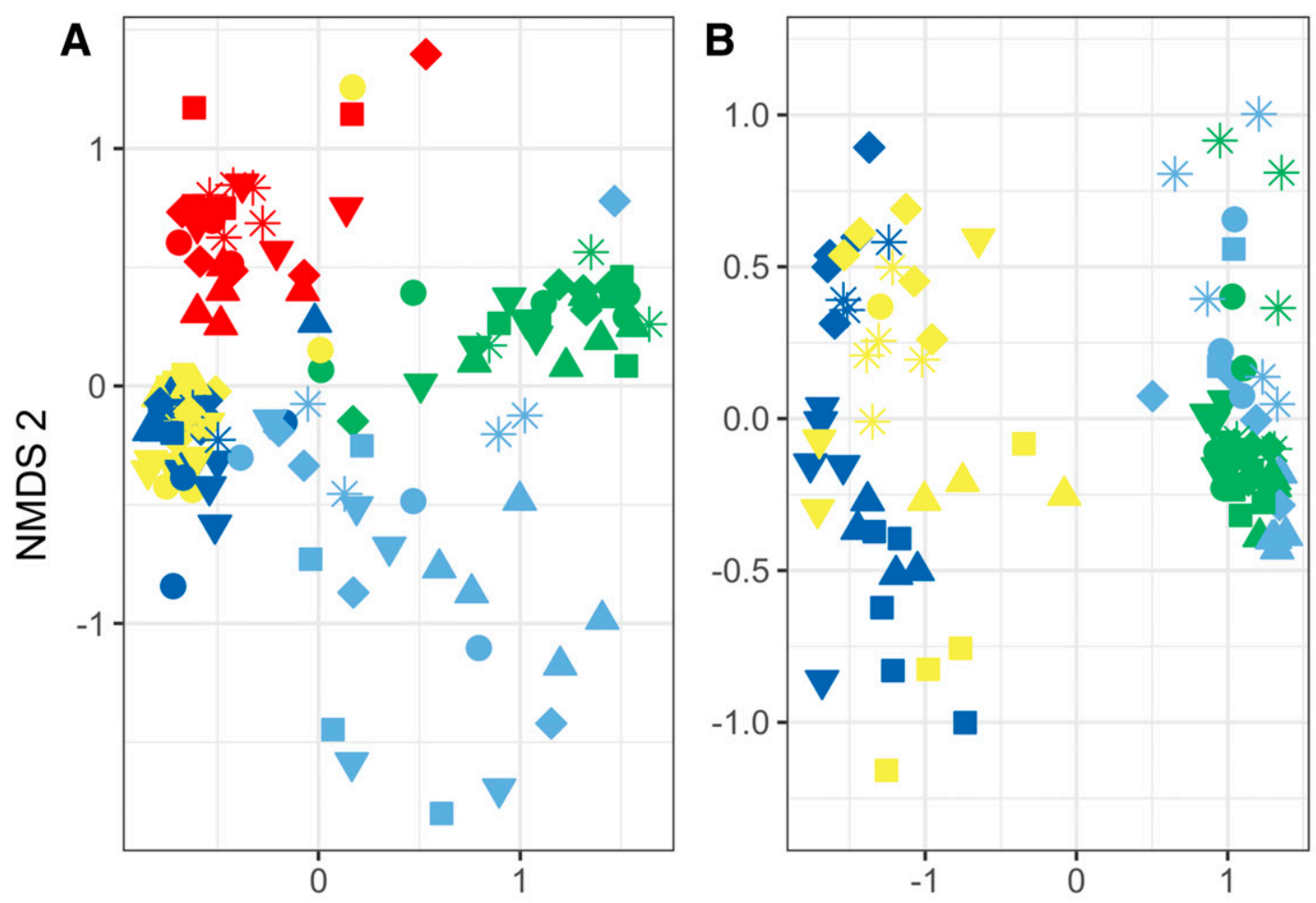

\section{Compartment}

- Bulk soil

Rhizosphere soil

- Root tissue

Leaves

Flowers

\section{NMDS 1}

Fig. 1. Both $\mathbf{A}$, bacterial (stress $=0.16$ ) and $\mathbf{B}$, fungal (stress $=0.11$ ) communities vary by plant compartment (bacterial: $R^{2}=0.32310, \mathrm{df}=4, P=0.001$; fungal: $R^{2}=0.47902, \mathrm{df}=3, P=0.001$ ), field location (bacterial: $R^{2}=0.08029$, df $=5, P=0.001$; fungal: $R^{2}=0.14361, \mathrm{df}=5, P=0.001$ ), and their interaction (bacterial: $R^{2}=0.16247, \mathrm{df}=20, P=0.001$; fungal: $R^{2}=0.15231$, $\mathrm{df}=13, P=0.001$ ). NMDS = nonmetric multidimensional scaling. Ordinations generated based on Bray-Curtis dissimilarity. 
Pearson's $r=0.0390, \mathrm{df}=4$; adjusted $P=1$; leaves: Pearson's $r=$ 0.2667 , df $=8$, adjusted $P=1$; flowers: Pearson's $r=0.1869, \mathrm{df}=1$, adjusted $P=1$ ).

Fungal taxa were favored differently across plant compartments (Fig. 3), with the most abundant classes ( $>1 \%$ of the fungal community) varying significantly in relative abundance across plant compartments (Kruskal-Wallis, all Bonferroni adjusted $P<0.001$ ). Notably, based on post hoc tests (Dunn tests, $P<0.05$ ), no class varied significantly in relative abundance between bulk and rhizosphere soils. Classes Sordariomycetes, Eurotiomycetes,
Mortierellomycetes, and Leotiomycetes were in greater relative abundance in bulk and rhizosphere soil communities relative to those of leaves or flowers. In contrast, classes Exobasidomycetes, Microbotryomycetes, and Tremellomycetes were more abundant on leaves and flowers. Isolates of Dothideomycetes were also more abundant on the leaves than any other plant compartment (Fig. 3).

The top five most abundant bacterial and fungal OTUs differ across plant compartments. We examined the top five most abundant fungal and bacterial OTUs within each plant compartment (Tables 2 and 3). We found that the portion of the community
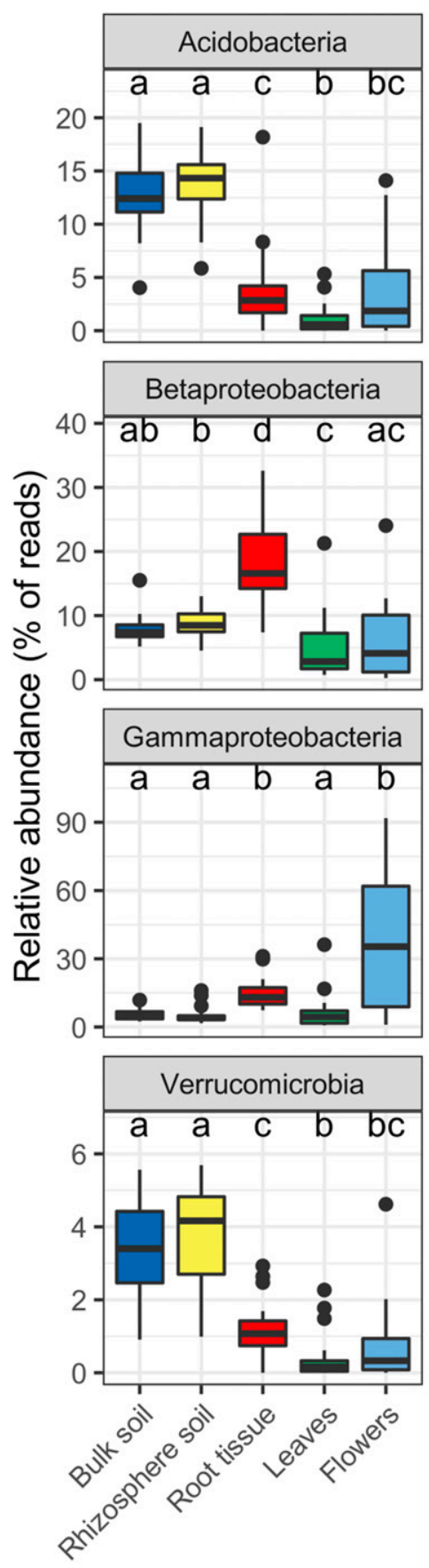
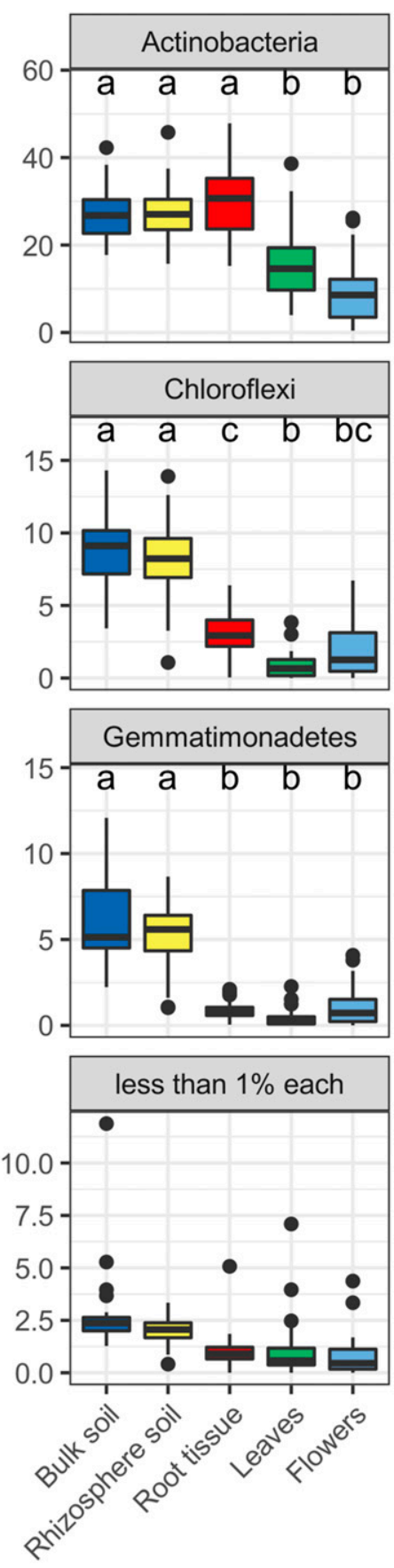
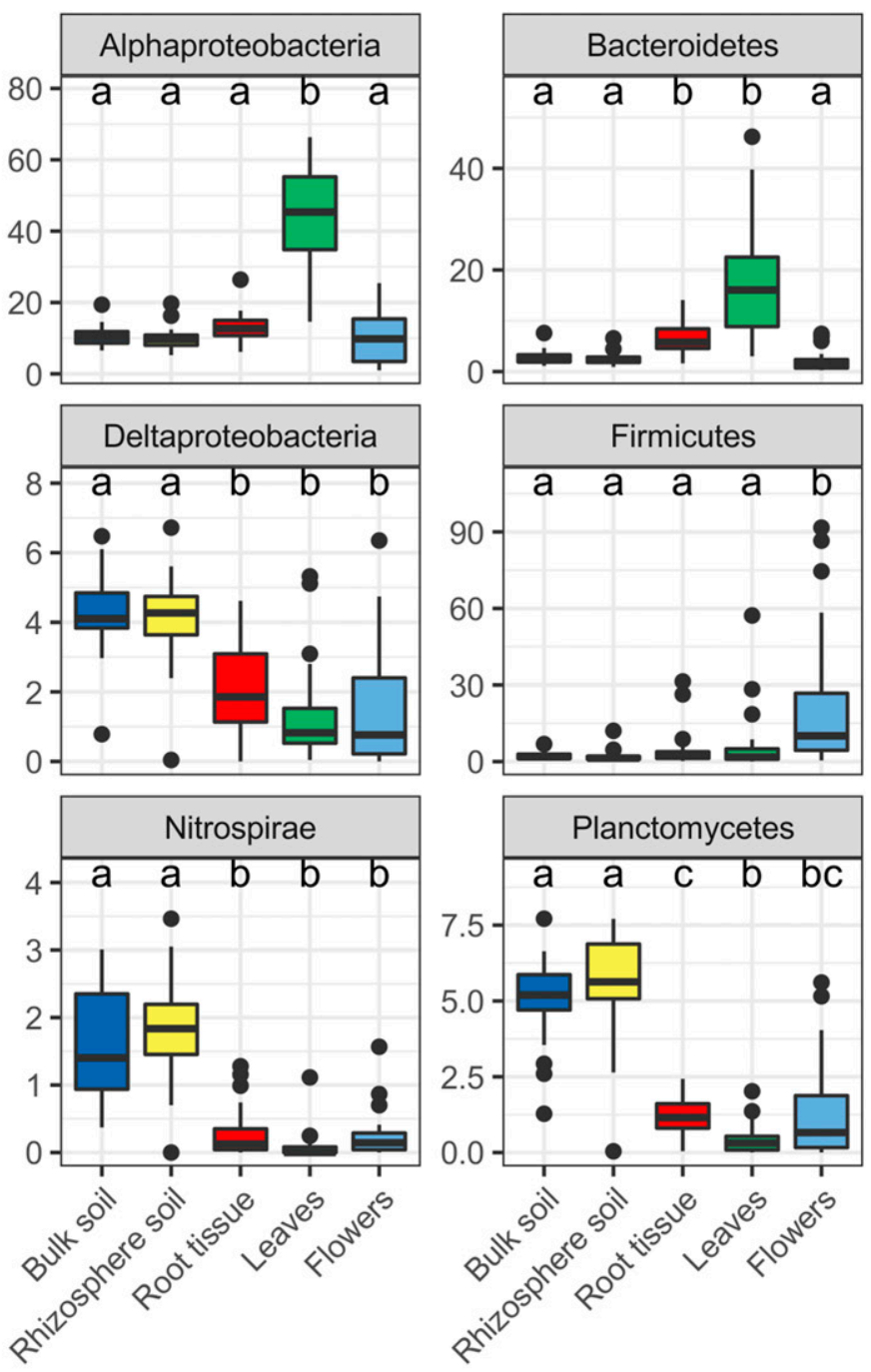

\section{Compartment}

Fig. 2. Relative abundances of all highly abundant bacterial phyla or classes ( $>1 \%$ of the rarefied community) differ significantly across plant compartments (Kruskal-Wallis with Bonferroni correction for multiple comparisons: all $P<0.05$ ). Proteobacteria operational taxonomic units (OTUs) have been grouped at the class level. For each phylum or class, all relative abundance values from the rarefied OTU table for each member OTU was summed for each sample. Post hoc Dunn test pairwise comparison grouping among plant compartment is indicated by lowercase letters $(P<0.05)$. 
represented by the top five OTUs differed across plant compartment. The top five most abundant bacterial OTUs in each compartment comprised approximately $7 \%$ of the bulk and rhizosphere soil communities and 19,47 , and $42 \%$ of the root tissue, leaf, and flower communities, respectively (Table 2). A similar trend was observed for the top five most abundant fungal OTUs, which comprised 38 and $37 \%$ of the bulk and rhizosphere soil communities, respectively, and approximately 74 and $77 \%$ of the leaf and flower communities, respectively (Table 3). Accordingly, Pielou's species evenness varied significantly across plant compartment for both bacterial and fungal communities (ANOVA; bacterial: $\mathrm{df}=4$, $F=60.657, P<0.001$; fungal: $\mathrm{df}=3, F=53.059, P<0.001)$. Leaves and flowers had lower bacterial and fungal evenness than bulk and rhizosphere soils. In addition, root tissues had lower bacterial evenness than bulk and rhizosphere soils but higher evenness than leaves or flowers (Fig. 4) (Post hoc Tukey; all $P<0.05$ ).

The top five most abundant bacterial OTUs found in root tissues, leaves, and flowers were all enriched significantly in these compartments compared with their relative abundance in bulk soil (DESeq2, $P<0.1$ ) (Table 2). In contrast, the most abundant
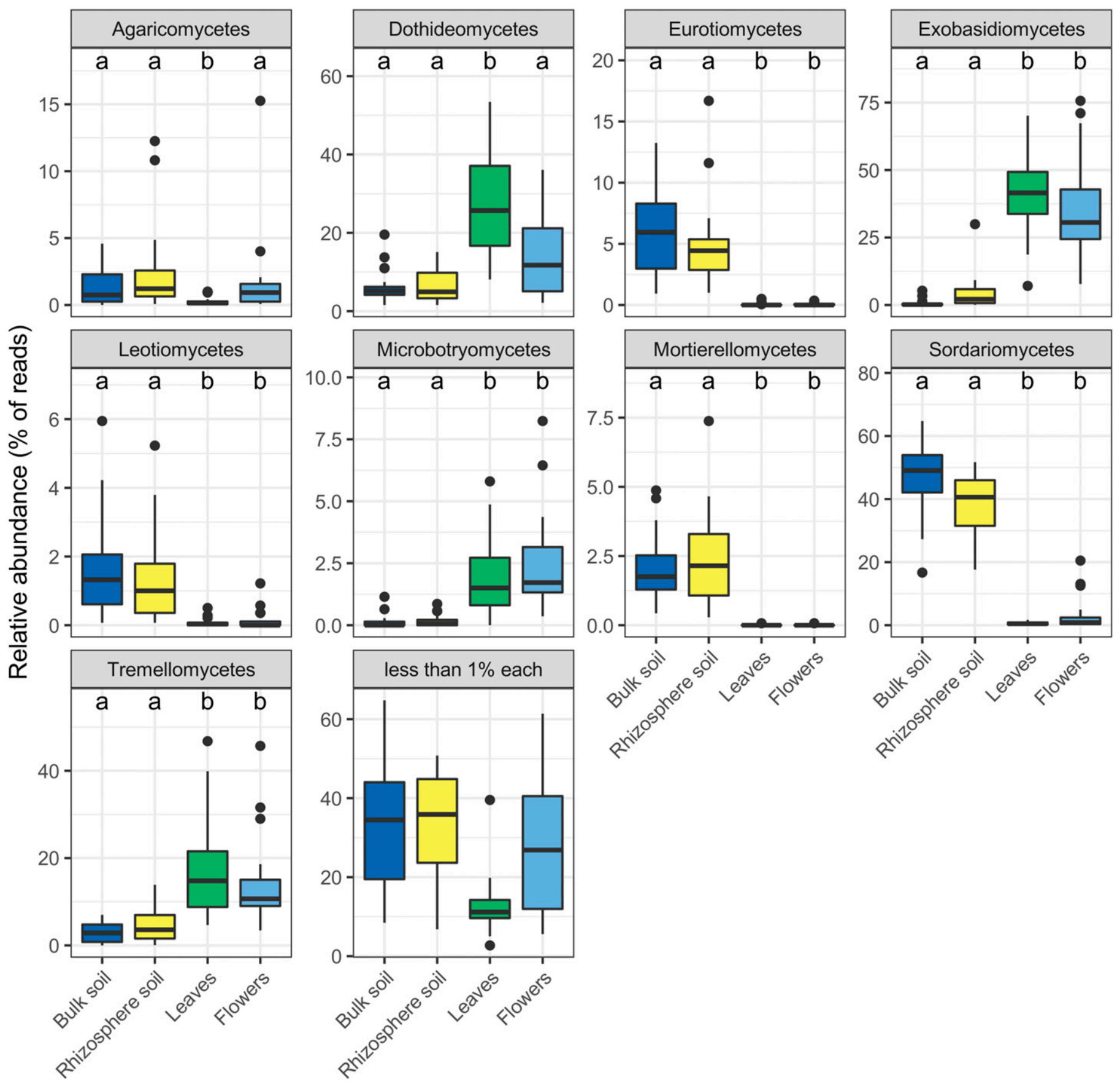

\section{Compartment}

Fig. 3. Relative abundances of all highly abundant fungal classes ( $>1 \%$ of the rarefied community) differ significantly across plant compartments (KruskalWallis with Bonferroni correction for multiple comparisons: all $P<0.05)$. For each class, all relative abundance values from the rarefied operational taxonomic unit (OTU) table for each member OTU was summed for each sample. Post hoc Dunn test pairwise comparison grouping among plant compartment is indicated by lowercase letters $(P<0.05)$. 
TABLE 2

Bacterial core operational taxonomic units (OTUs) (Core?), identified as ubiquitous in each plant compartment (Ubiq plant) and enriched in the plant samples compared with bulk soil $\left(\log _{2} \mathrm{FC}\right)$, as well as the top five most abundant OTUs (Rank) within each compartment ${ }^{a}$

\begin{tabular}{|c|c|c|c|c|c|c|c|}
\hline Compartment (samples), OTU ${ }^{\mathrm{b}}$ & Abundance (SE) $(\%)^{\mathrm{c}}$ & $\log _{2} \mathrm{FC}(\mathrm{SE})$ & Rank & Ubiq plant & Ubiq soil & Core? & Best classification (level) \\
\hline \multicolumn{8}{|l|}{ Bulk soil (26) } \\
\hline OTU.10 & $1.77(0.25)$ & NA & 1 & NA & Yes & No & Pseudarthrobacter (genus) \\
\hline OTU.21 & $1.29(0.13)$ & NA & 3 & NA & Yes & No & Janibacter (genus) \\
\hline OTU.66 & $1.23(0.23)$ & NA & 4 & NA & Yes & No & Comamonadaceae (family) \\
\hline OTU.39 & $1.12(0.14)$ & NA & 5 & NA & Yes & No & Lysobacter (genus) \\
\hline OTU.10 & $2.00(0.22)$ & NS & 1 & Yes & Yes & No & Pseudarthrobacter (genus) \\
\hline OTU.28 & $1.63(0.12)$ & NS & 2 & No & No & No & Chloroflexi, KD4-96 (class) \\
\hline OTU.21 & $1.20(0.16)$ & NS & 3 & No & Yes & No & Janibacter (genus) \\
\hline OTU.66 & $1.00(0.11)$ & NS & 4 & Yes & Yes & No & Comamonadaceae (family) \\
\hline OTU.63 & $0.85(0.07)$ & NS & 5 & No & No & No & Acidobacteria, subgroup 6 (class) \\
\hline OTU.49 & $3.47(0.65)$ & $2.46(0.32)$ & 3 & Yes & Yes & Yes & Comamonadaceae (family) \\
\hline OTU.18 & $2.68(0.60)$ & $1.53(0.29)$ & 4 & Yes & Yes & Yes & Massilia (genus) \\
\hline OTU.117 & $2.43(0.45)$ & $2.75(0.35)$ & 5 & Yes & Yes & Yes & Aquabacterium (genus) \\
\hline OTU.74 & $1.09(0.23)$ & $2.69(0.46)$ & 14 & Yes & No & Yes & Rhizobium (genus) \\
\hline OTU.91 & $1.05(0.35)$ & $2.62(0.52)$ & 17 & Yes & No & Yes & Pseudomonas (genus) \\
\hline OTU.8 & $0.59(0.12)$ & $2.92(0.33)$ & 32 & Yes & Yes & Yes & Sphingomonas (genus) \\
\hline \multicolumn{8}{|l|}{ Leaves (28) } \\
\hline OTU.8 & $13.14(1.88)$ & $6.70(0.39)$ & 1 & Yes & Yes & Yes & Sphingomonas (genus) \\
\hline OTU.5 & $12.81(1.99)$ & $7.65(0.46)$ & 2 & Yes & No & Yes & Sphingomonas (genus) \\
\hline OTU.9 & $8.55(1.73)$ & $7.46(0.57)$ & 3 & Yes & No & Yes & Methylobacterium (genus) \\
\hline OTU.1896 & $0.23(0.04)$ & $4.60(0.53)$ & 44 & Yes & No & Yes & Sphingomonas (genus) \\
\hline \multicolumn{8}{|l|}{ Flowers (26) } \\
\hline OTU.7 & $12.56(3.19)$ & $6.21(0.59)$ & 1 & No & No & No & Pseudomonas (genus) \\
\hline OTU.4 & $11.67(4.41)$ & $9.92(0.72)$ & 2 & No & No & No & Lactococcus lactis (species) \\
\hline OTU.12 & $6.33(2.01)$ & $8.03(0.73)$ & 3 & No & No & No & Pantoea (genus) \\
\hline OTU.20 & $6.23(2.00)$ & $5.85(0.62)$ & 4 & No & No & No & Enterobacter (genus) \\
\hline OTU.6 & $5.53(3.59)$ & $2.06(0.56)$ & 5 & Yes & Yes & Yes & Bacillus cereus (species) \\
\hline OTU.13 & $2.85(0.85)$ & $2.02(0.32)$ & 7 & Yes & Yes & Yes & Ralstonia (genus) \\
\hline OTU.61 & $1.22(0.55)$ & $1.52(0.41)$ & 10 & Yes & Yes & Yes & Bacillus (genus) \\
\hline OTU.5 & $1.05(0.36)$ & $4.94(0.55)$ & 11 & Yes & No & Yes & Sphingomonas (genus) \\
\hline OTU.8 & $1.01(0.33)$ & $4.07(0.46)$ & 12 & Yes & Yes & Yes & Sphingomonas (genus) \\
\hline OTU.9568 & $0.72(0.18)$ & $6.74(0.56)$ & 16 & Yes & No & Yes & Bradyrhizobium (genus) \\
\hline OTU.9 & $0.37(0.13)$ & $3.41(0.54)$ & 24 & Yes & No & Yes & Methylobacterium (genus) \\
\hline
\end{tabular}

a The number of samples used for these analyses differed across plant compartments due to removal of samples with low sequence counts. Relative abundance, as percentage of rarefied reads, was averaged across all samples. Column $\log _{2}$ FC indicates the $\log _{2}$-fold change in read count between plant compartment and bulk soil for OTUs that were enriched significantly (Benjamini and Hochberg adjusted $P<0.1$ ), calculated with DESeq2 on unrarefied read counts. NS indicates nonstatistically significant enrichment. NA indicates measures that are not applicable for the bulk soil. Bold values are used for emphasis. OTU taxonomy is given as the highest classification level determined from the SILVA database.

b Plant compartment (number of samples) and OTU ID.

c Percent relative abundance and standard error (SE). 
bacterial OTUs in the bulk and rhizosphere soil communities were highly similar, and none of the top-abundance rhizosphere taxa were enriched significantly relative to bulk soil (DESeq2, $P>0.1$ ) (Table 2). Of the OTUs observed in root tissue, the most enriched was a member of the family Halieaceae (OTU.17; $\log _{2}$-fold change $=8.62 \pm 0.72$ ). This OTU had very low abundance in the bulk soil (and was below the detection limit in multiple samples) but was the second most abundant within root tissue $(4.94 \pm 1.33 \%)$. Similarly, an OTU classified as Lactococcus lactis (OTU.4) was highly enriched on the flowers compared with soil ( $\log _{2}$-fold change $=9.92 \pm 0.72)$ and highly abundant on the flowers $(11.67 \pm$ $4.41 \%)$.

The top five most abundant fungal OTUs found in leaves were also enriched significantly compared with their relative abundance

TABLE 3

Fungal core operational taxonomic units (OTUs) (Core?), identified as ubiquitous in each plant compartment (Ubiq plant) and enriched in the plant samples compared with bulk soil $\left(\log _{2} \mathrm{FC}\right)$, as well as the top five most abundant OTUs (Rank) within each compartment ${ }^{\mathrm{a}}$

\begin{tabular}{|c|c|c|c|c|c|c|c|}
\hline Compartment (samples),OTU & Abundance (SE) $(\%)^{c}$ & $\log _{2} \mathrm{FC}(\mathrm{SE})$ & Rank & Ubiq plant & Ubiq soil & Core? & Best classification (level) \\
\hline \multicolumn{8}{|l|}{ Bulk soil (22) } \\
\hline OTU_13 & $13.97(2.28)$ & NA & 1 & NA & Yes & NA & Verticillium dahlia (species) \\
\hline OTU_34 & $6.84(1.50)$ & NA & 3 & NA & Yes & NA & Fungi (kingdom) \\
\hline OTU_309 & $5.08(1.52)$ & NA & 4 & NA & Yes & NA & Fungi (kingdom) \\
\hline OTU_13 & $10.94(1.60)$ & NS & 1 & Yes & Yes & No & V. dahlia (species) \\
\hline OTU_34 & $9.57(1.81)$ & NS & 2 & Yes & Yes & No & Fungi (kingdom) \\
\hline OTU_8 & $7.63(1.57)$ & NS & 3 & Yes & Yes & No & Fusarium (genus) \\
\hline OTU_21 & $4.66(1.45)$ & $2.85(0.50)$ & 4 & No & No & No & Tilletiopsis washingtonensis (species) \\
\hline OTU_21 & $36.25(3.11)$ & $5.88(0.42)$ & 1 & Yes & No & Yes & T. washingtonensis (species) \\
\hline OTU_174 & $16.75(1.67)$ & $5.58(0.42)$ & 2 & Yes & No & Yes & Epicoccum (genus) \\
\hline OTU_27 & $11.64(1.64)$ & $6.42(0.41)$ & 3 & Yes & No & Yes & B. alba (species) \\
\hline OTU_115 & $5.27(0.68)$ & $2.09(0.25)$ & 4 & Yes & Yes & Yes & Ascomycota (phylum) \\
\hline OTU_125 & $4.15(0.59)$ & $3.37(0.44)$ & 5 & Yes & No & Yes & Fungi (kingdom) \\
\hline OTU_84 & $2.21(0.44)$ & $5.72(0.61)$ & 8 & Yes & No & Yes & Neoascochyta (genus) \\
\hline OTU_118 & $1.74(0.33)$ & $5.91(0.50)$ & 9 & Yes & No & Yes & Alternaria infectoria (species) \\
\hline OTU_331 & $0.88(0.12$ & $6.38(0.51)$ & 10 & Yes & No & Yes & Dioszegia hungarica (species) \\
\hline OTU_338 & $0.80(0.12)$ & $6.21(0.53)$ & 11 & Yes & No & Yes & Basidiomycota (phylum) \\
\hline OTU_21 & $32.30(5.16)$ & NS & 1 & Yes & No & No & T. washingtonensis (species) \\
\hline OTU_115 & $22.33(3.35)$ & NS & 2 & Yes & Yes & No & Ascomycota (phylum) \\
\hline OTU_27 & $10.80(2.24)$ & $2.07(0.49)$ & 3 & Yes & No & Yes & B. alba (species) \\
\hline OTU_174 & $8.18(1.99)$ & NS & 4 & Yes & No & No & Epicoccum (genus) \\
\hline OTU_3520 & $3.22(2.26)$ & NS & 5 & No & No & No & T. washingtonensis (species) \\
\hline OTU_333 & $1.43(0.31)$ & $2.53(0.68)$ & 8 & Yes & No & Yes & S. ruberrimus (species) \\
\hline
\end{tabular}

a The number of samples used for these analyses differed across plant compartments due to removal of samples with low sequence counts. Relative abundance, as percent of rarefied reads, was averaged across all samples. Column $\log _{2}$ FC indicates the $\log _{2}$-fold change in read count between plant compartment and bulk soil for OTUs that were enriched significantly (Benjamini and Hochberg adjusted $P<0.1$ ), calculated with DESeq2 on unrarefied read counts. NS indicates nonstatistically significant enrichment. NA indicates measures that are not applicable for the bulk soil. Bold values are used for emphasis. OTU taxonomy is given as the highest classification level determined from the UNITE database.

b Plant compartment (number of samples) and OTU ID.

c Percent relative abundance and standard error (SE). 
in bulk soil; however, only one from the flowers was enriched significantly. The top five most abundant fungal OTUs had similar abundance in bulk and rhizosphere soils, with only one highabundance rhizosphere taxon, OTU_21 (classified as Tilletiopsis washingtonensis), enriched significantly in the rhizosphere relative to bulk soil $\left(\log _{2}\right.$-fold change $\left.=2.85 \pm 0.50\right)$ (Table 3$)$. Of the top five most abundant fungal OTUs found on the leaves and flowers, four are shared between the two plant compartments. These include OTUs matching T. washingtonensis (OTU_21) which is found widely throughout the hemp samples, an OTU matching Bullera alba (OTU_27), an unclassified Epicoccum sp. (OTU_174), and an unclassified Ascomycota sp. (OTU_115).

The core microbiome of hemp. We looked for both bacterial and fungal OTUs that may be part of a core microbiome of hemp. We defined a member of the core microbiome for a given plant compartment as an OTU that was ubiquitous across all plants in all sampling locations and enriched significantly in their plant compartment relative to the bulk soil.

We identified 20 candidate core bacterial OTUs across our hemp plants, all of which were identified in the root tissue, on the leaf surface, or in the flowers (Table 2). There were six core bacterial OTUs from the root tissue, all of which were members of the phylum Proteobacteria. There were 11 core bacterial OTUs from the leaf surface, made up of members of the Proteobacteria, Actinobacteria, and Bacteroidetes. In all, 9 of the 11 most abundant OTUs on the leaves were part of this core group. The two most abundant of these OTUs (OTU.8 and OTU.5, representing $13.14 \pm$ 1.88 and $12.81 \pm 1.99 \%$ of the leaf bacterial community, respectively) were classified in the genus Sphingomonas. There were seven core bacterial OTUs from the flowers, made up of members of the Proteobacteria and Firmicutes. Three of the core flower OTUs were also core leaf OTUs (OTU.5, OTU.8, and OTU.9). OTU.8 was a core OTU in the root tissue, leaf surface, and flower. None of the ubiquitous bacterial OTUs in the rhizosphere were enriched significantly in the rhizosphere compared with the bulk soil.

When we applied our core microbiome definition to fungal OTUs, we found 14 candidate core OTUs (Table 3). All core fungal OTUs were classified as Basidiomycota or Ascomycota, or unclassified at the phylum level. We found only one core fungal OTU in the rhizosphere soil, OTU_27, classified as B. alba. This OTU was also a core OTU on the leaves and in the flowers. There were 14 core fungal OTUs found on the leaves, including all of the top 5 most abundant OTUs. By far the most abundant core fungal OTU on the leaves was OTU_21, classified as T. washingtonensis, making up $36.25 \pm 3.11 \%$ of the leaf fungal community. This OTU was also highly abundant $(32.30 \pm 5.16 \%)$ and ubiquitous in the flowers, though not significantly enriched in that compartment compared with the bulk soil. There were only two core fungal OTUs found in the flowers, both of which were also core on the leaves.

Bacteria and fungi enriched significantly in the rhizosphere compared with bulk soil. We found little evidence for a core hemp rhizosphere microbiome, with only one core fungal OTU identified. Furthermore, for the most part, the top five most abundant bacterial and fungal OTUs were shared between the rhizosphere and bulk soil (Tables 1 and 2). To expand our search for bacterial and fungal OTUs that may be enriched in the hemp rhizosphere, we used DESeq2 to look for OTUs that were enriched significantly relative to the bulk soil but not necessarily ubiquitous or highly abundant in the rhizosphere. We found only one bacterial and two fungal OTUs enriched significantly in the rhizosphere. The bacterial OTU was classified in the family Pseudonocardiaceae (OTU.79; $\log _{2}$-fold change $=1.49 \pm 0.29, P=0.06)$. The two fungal OTUs matched T. washingtonensis (OTU_21; $\log _{2}$-fold change $=2.85 \pm 0.50$,
$P=2.65 \mathrm{E}-5)$ and $B$. alba $\left(\mathrm{OTU} \_27\right.$; DESeq $2 \log _{2}$-fold change $=$ $2.43 \pm 0.41, P=2.65 \mathrm{E}-5)$. These two fungal OTUs were also identified within the core microbiome of leaves and flowers.

\section{DISCUSSION}

Characterizing the microbes associated with an economically valuable crop is an important first step in understanding how the interactions between the plant and its microbiome influence crop yield and health. Here, we examined the bacteria and fungi

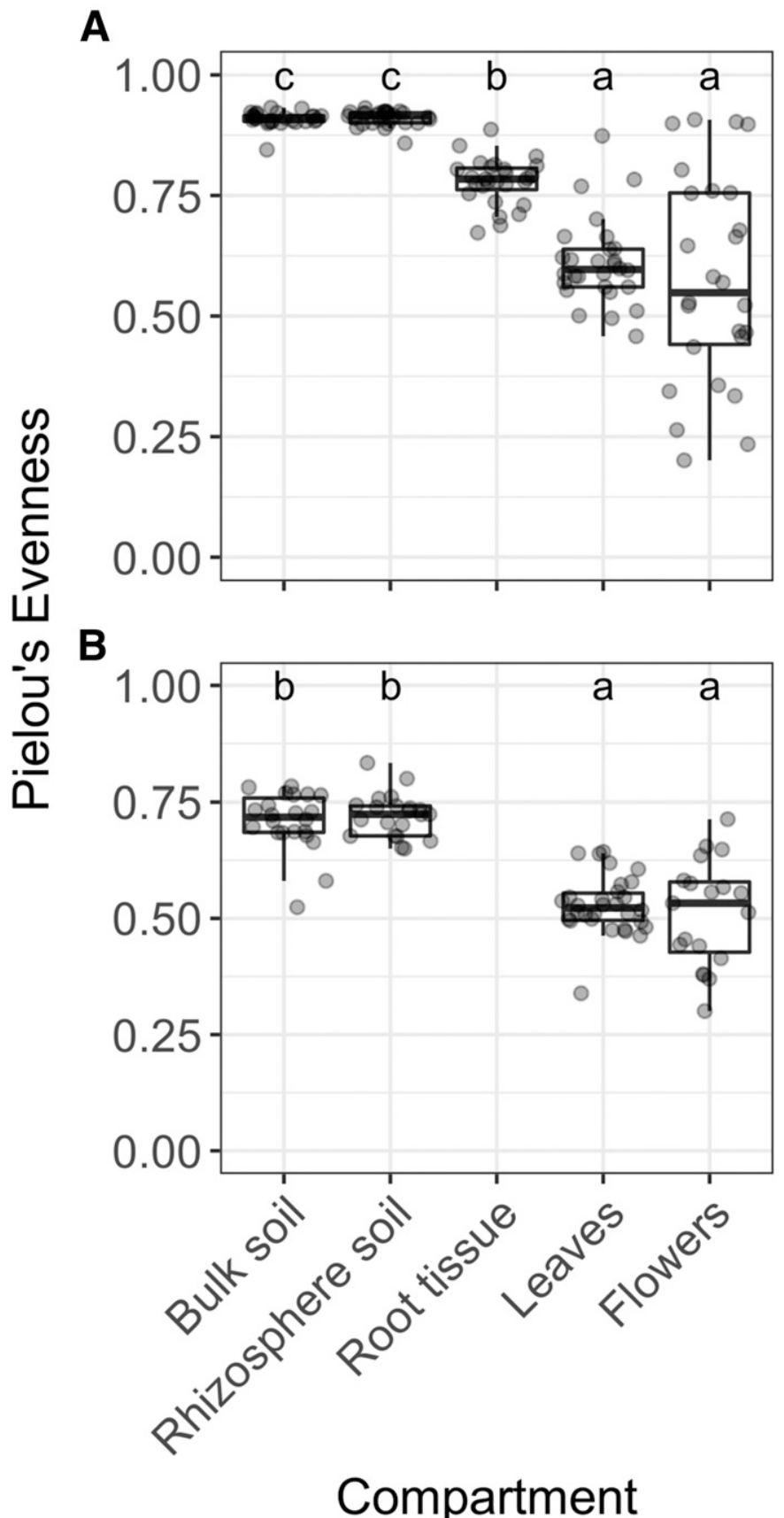

Fig. 4. Pielou's evenness for A, bacterial and B, fungal communities varies significantly across plant compartments (analysis of variance; bacterial: $\mathrm{df}=4, F$ statistic $=60.657, P<0.001$; fungal: $\mathrm{df}=3, F$ statistic $=$ 53.059, $P<0.001$ ). Post hoc Tukey tests show that, in both cases, leaves and flowers have lower species evenness than bulk and rhizosphere soils. Pairwise comparison grouping among plant compartment is indicated by lowercase letters. 
associated with the rhizosphere, root tissue, leaf surface, and flowers of the hemp cultivar Anka. We identified the dominant taxa across plant compartments and characterized the core microbiome of this cultivar. We found that plant compartment (rhizosphere, root, leaf, or flower) had strong effects on the bacterial and fungal taxa present (Figs. 1, 2, and 3). We observed that the OTUs most highly abundant in the roots, leaves, and flowers occurred at low abundance in soils, suggesting selective enrichment within these plant compartments (Tables 1 and 2). The top five most abundant taxa also made up a greater proportion of the community in the roots, leaves, and flowers than the top most abundant taxa in the bulk or rhizosphere soils. This observation suggests that the root tissue and the phyllosphere are dominated by few highly abundant OTUs, which was further confirmed by the low evenness in these plant compartments (Fig. 4). Reduced microbial community evenness in plant compartments has been documented in other plant species (Bodenhausen et al. 2013).

We also found that field site explained significant variation in community composition within each plant compartment. The field site effect was strongest for bulk and rhizosphere soil communities. It is likely that a number of factors drive this site-specific variation, particularly soil chemistry. Although we did not have the data resolution to tease apart how soil chemistry may influence aboveand belowground microbial communities associated with hemp, we note that most of our sites differed in their soil type (Table 1). Our knowledge of the hemp microbiome would benefit from studies linking environmental factors to microbial community composition, especially factors related to management practices such as tillage and fertilization. Altogether, these findings suggest that, although local environmental factors can influence the hemp microbiome, certain microbes are highly enriched in hemp regardless of field site. We did not observe any relationship between bacterial or fungal community composition and crop yield across field sites.

We found little evidence for a core microbiome in the hemp rhizosphere when we examined either the top five most abundant OTUs or ubiquitous OTUs in the rhizosphere soils. Only one fungal OTU was determined to be a core OTU by our definition (OTU_27). We expanded our search to look at all OTUs that were enriched significantly in the rhizosphere soil compared with the bulk soil. Only one bacterial OTU and two fungal OTUs were enriched significantly in the rhizosphere when compared with bulk soil. OTU.79 was classified as an Actinobacteria within the family Pseudonocardiaceae and, when aligned to the NCBI RefSeq database, matched genus Lechevalieria (BLAST, 100\% identity). This genus includes species commonly observed in soil and rhizosphere habitats. The two fungal OTUs (OTU.21 and OTU.27) enriched in the rhizosphere were classified as T. washingtonensis and B. alba, respectively. These two OTUs were also ubiquitous and enriched significantly, relative to bulk soil, on hemp leaves (both OTUs) and flowers (OTU_27). These observations suggest that these fungi have high affinity for hemp. Most bacterial and fungal OTUs that were observed widely in the hemp rhizosphere were also ubiquitous in the bulk soil and were not enriched in rhizosphere relative to the bulk soil (Tables 2 and 3). A number of the microbes that were highly abundant in the rhizosphere belong to taxa commonly found in plants; however, we only included in the core hemp microbiome those OTUs that were enriched significantly in the hemp rhizosphere relative to bulk soil. Altogether, our results suggest that hemp does not have a strong immediate effect on overall microbial community composition in the rhizosphere. This result is surprising because plants are widely known to significantly influence their rhizosphere microbial community (Berendsen et al. 2012). However, environmental factors (Peiffer et al. 2013), particularly agricultural practices (Schmidt et al. 2019), influence strength of plant effect on rhizosphere microbiome composition. Other cultivars of C. sativa have previously displayed weak effects on their rhizosphere microbiomes, suggested to be due to root decay (Winston et al. 2014). Alternative reasons for an apparent weak plant effect may include the plant development stage at sampling or root structure and rhizosphere sampling method. Future studies should more closely examine the influence of hemp on rhizosphere microbial composition, including analysis of root exudates and community succession over hemp growth stages.

T. washingtonensis is a widespread yeast-like fungus often isolated from the phyllosphere of plants (Urquhart and Punja 2002). It has been studied as a biocontrol agent against powdery mildew (Urquhart and Punja 2002; Urquhart et al. 1994); however, it has also been suggested as the causative agent of white haze on apple fruit (Baric et al. 2010). B. alba is another widespread yeast-like fungus with at least one isolate found to produce antifungal compounds (Golubev et al. 1997). Although these fungi have not been studied in the context of hemp, their rhizosphere enrichment and characterization as dominant members of the microbiome of hemp leaves and flowers makes them intriguing targets for further study in this system. Their potential as biocontrol agents of common diseases such as powdery mildew, a disease present on $C$. sativa (McPartland and Cubeta 1997; Weldon et al. 2020), may implicate these fungi as factors of disease resistance in this crop.

In our characterization of the core root microbiome of hemp, we found evidence for further plant growth or health-promoting microbes in close association with the plants. From the root tissue, we found six core OTUs. One of these, OTU.49, matched species within the family Comamonadaceae, genus Variovorax (BLAST to NCBI RefSeq, 100\% identity with NR_114214.1, NR_169353.1, NR_169352.1, NR_041588.1, and NR_112562.1). Members of this genus are common in soils and some species are associated with plant growth promotion (Han et al. 2011; Schmalenberger et al. 2008). OTU.18 was classified within the Oxalobacteraceae genus Massilia. Species of this genus have been implicated as mediators of plant growth promotion in numerous plant species (Ofek et al. 2012). OTU.117, classified to the Burkholderiales genus Aquabacterium, have been detected in high abundance in the rhizosphere of numerous plant species (Bai et al. 2020; Schmalenberger and Tebbe 2003; Wang et al. 2014) as well as ground and drinking water (Guo et al. 2019; Kalmbach et al. 2000). Similarly, relatives of the other three core root OTUs classified to genera Rhizobium, Pseudomonas, or Sphingomonas have been widely found to be associated with plants in various ways. Although not a core bacterium by our definition because it was not ubiquitous in hemp roots, OTU.17, classified within the family Halieaceae, was very highly enriched in the root tissue of many hemp plants. Its high enrichment in this particular plant compartment suggests an interaction between the bacterium and hemp roots. To the best of our knowledge, little is known about members of this family in terms of plant interactions and, therefore, further study of this group may be of particular interest. Due to a limitation of experimental design, we were unable to examine fungi in close association with the root tissue; however, future studies using methods to remove host DNA prior to sequencing may identify fungi in close association with hemp roots.

Our analysis of the core phyllosphere bacterial OTUs of hemp revealed well-known phyllosphere organisms, including microbes known to promote plant growth and disease resistance. Of the 11 core leaf bacterial OTUs, the top five most abundant were classified to the genera Sphingomonas, Methylobacterium, and Hymenobacter. These genera are widely observed in plant phyllospheres (Delmotte et al. 2009; Grady et al. 2019; Rastogi et al. 2013; Turner et al. 2013; Vorholt 2012; Wellner et al. 2011). Certain 
Sphingomonas strains have been observed to confer resistance to the leaf pathogen Pseudomonas syringae (Innerebner et al. 2011). Similarly, numerous studies have observed that Methylobacterium spp. can promote plant growth and yield as a result of methanol cycling (Abanda-Nkpwatt et al. 2006) and the production of auxins (Ivanova et al. 2001), cytokinins (Ivanova et al. 2000), and vitamins (Trotsenko et al. 2001).

The community composition on hemp flowers varied widely among plants. The hemp flower is a dynamic environment, with structural and chemical changes occurring as the plant ages and interacts with pollinators. This dynamic environment may play a role in the high variability observed across plants. Despite this variability, we identified seven core bacterial OTUs in the flower. Some taxa discussed previously included the genera Sphingomonas and Methylobacterium. We also found two members of the genus Bacillus, one of which was classified to the species Bacillus cereus. Although this genus is widespread in soil and plant microbiomes, $B$. cereus strains found in the phyllosphere have been shown to promote plant growth and pathogen resistance (Kloepper et al. 2004; Saleem et al. 2017). OTU.13 was classified to the genus Ralstonia, a notable genus for containing a number of prominent plant pathogens. This particular OTU, however, matches isolates of Ralstonia pickettii (BLAST to NCBI RefSeq, 100\% identity with NR_114126.1, NR_043142.1, and NR_113352.1). Strains of this species have been proposed as a biocontrol agent against diseasecausing Ralstonia spp. (Wei et al. 2013). Although not a core OTU by our definition, one of the bacterial OTUs with the greatest enrichment in the flower samples relative to the bulk soil was OTU.4, classified as Lactococcus lactis. The genus Lactococcus includes fermentative aerotolerant anaerobes, which are often observed on plants and which are essential for the production of fermented plant products (Di Cagno et al. 2013). Because many of the phytochemicals of interest (e.g., cannabinoids) from hemp are produced in the flowers, these highly abundant Lactococcus spp. as well as the core bacterial OTUs discussed here may play a role in phytochemical yield and quality.

Most fungal OTUs of interest in the phyllosphere were shared between leaves and flowers. These shared core fungal OTUs included T. washingtonensis and Bullera alba (discussed previously). A leaf core fungal member, OTU_174, classified to the genus Epicoccum, was also highly abundant and ubiquitous in the flower community, though not significantly enriched in that compartment. The genus Epicoccum is often observed in soils and in the plant phyllosphere and some strains promote disease resistance in grapevines (Del Frari et al. 2019). OTU_118, a core fungal OTU on the leaf, was classified as Alternaria infectoria (OTU_118). This species, along with close relatives, has been shown to cause diseases such as leaf blight in wheat and other grains (Perelló and Sisterna 2006; Prasada and Prabhu 1962). A. infectoria can also cause cutaneous diseases in humans, usually associated with immunocompromised individuals (Dubois et al. 2005; Lopes et al. 2013). The effect of this fungal species on hemp or hemp products has yet to be determined; however, isolates of the Alternaria genus have been isolated from hemp cultivar Anka leaves in other studies (Scott et al. 2018). We also identified a core leaf fungal OTU classified to the genus Neoascochyta (OTU_84). Members of this genus are widely found associated with plants and include plant pathogens, particularly for grasses (Chen et al. 2017; Golzar et al. 2019).

Our results show that numerous microbes are enriched within the roots, leaves, and flowers of hemp. Many of the microbes enriched on hemp plants are observed widely on various plant species and are linked to plant growth promotion and disease resistance, while a few have been implicated as possible plant pathogens, though not necessarily in $C$. sativa. Future research should include more
C. sativa cultivars to understand cultivar-specific variability of the microbiome. Rhizosphere microbiome variability has previously been observed across cultivars of $C$. sativa with high THC content (Winston et al. 2014) and those of varying THC and cannabidiol levels (Comeau et al. 2020). Field trials connecting the microbiome communities and crop yields or disease resistance should also be a focus for future research.

The microbes found within plant compartments and the mechanisms by which plants enrich for their microbiomes vary significantly across time and across plant growth stages (Copeland et al. 2015; Walters et al. 2018). The rhizosphere and endosphere of C. sativa cultivars have been shown to vary across plant growth stages as well (Comeau et al. 2020). Because our study determined microbiome composition at flowering, we may be missing core hemp microbiome members important at other stages. Future studies aimed at further elucidating the core hemp microbiome would benefit from more time points at other key stages of growth.

As we show here, some members of the hemp microbiome are similar to those that promote growth in other plant species. Specifically, understanding how the microbiome composition influences crop yield parameters such as fiber or grain quantity or quality and phytochemical production may lead to better cultivation practices. Modulation to the hemp microbiome to aid in fiber processing, specifically retting, has been proposed (Law et al. 2020). Similarly, microbes found on the hemp flowers may play a role in quality of the phytochemicals (Taghinasab and Jabaji 2020) and may be important for preventing contamination by opportunistic human pathogens. Identifying microbiome members with specific disease resistance capabilities allows for development of new sustainable biocontrol agents. As cultivation of hemp becomes more widespread, there will be many opportunities to study and improve upon our understanding of the diverse microbiome of this valuable crop.

\section{ACKNOWLEDGMENTS}

We thank S. Higgins for advice on development of the fungal ITS1 processing pipeline and H. Lange and M. Bekauri for technical assistance in collecting and sampling hemp plants.

\section{LITERATURE CITED}

115th Congress. 2018. H.R.2 - Agriculture Improvement Act of 2018. https:// www.congress.gov/bill/115th-congress/house-bill/2

Abanda-Nkpwatt, D., Müsch, M., Tschiersch, J., Boettner, M., and Schwab, W. 2006. Molecular interaction between Methylobacterium extorquens and seedlings: Growth promotion, methanol consumption, and localization of the methanol emission site. J. Exp. Bot. 57:4025-4032.

Bai, Y., Ren, P., Feng, P., Yan, H., and Li, W. 2020. Shift in rhizospheric and endophytic bacterial communities of tomato caused by salinity and grafting. Sci. Total Environ. 734:139388.

Baric, S., Lindner, L., Marschall, K., and Dalla Via, J. 2010. Haplotype diversity of Tilletiopsis spp. causing white haze in apple orchards in Northern Italy. Plant Pathol. 59:535-541.

Barnett, S. 2020. seb369/hemp_microbiome: First release of hemp microbiome project data. https://zenodo.org/record/4001760

Bengtsson-Palme, J., Ryberg, M., Hartmann, M., Branco, S., Wang, Z., Godhe, A., De Wit, P., Sánchez-García, M., Ebersberger, I., de Sousa, F., Amend, A. S., Jumpponen, A., Unterseher, M., Kristiansson, E., Abarenkov, K., Bertrand, Y. J. K., Sanli, K., Eriksson, K. M., Vik, U., Veldre, V., and Nilsson, R. H. 2013. Improved software detection and extraction of ITS1 and ITS2 from ribosomal ITS sequences of fungi and other eukaryotes for analysis of environmental sequencing data. Methods Ecol. Evol. 4: 914-919.

Berendsen, R. L., Pieterse, C. M. J., and Bakker, P. A. H. M. 2012. The rhizosphere microbiome and plant health. Trends Plant Sci. 17: 478-486. 
Bodenhausen, N., Horton, M. W., and Bergelson, J. 2013. Bacterial communities associated with the leaves and the roots of Arabidopsis thaliana. PLoS One 8: e56329.

Boller, T. 1995. Chemoperception of microbial signals in plant cells. Annu. Rev. Plant Physiol. 46:189-214.

Busby, P. E., Soman, C., Wagner, M. R., Friesen, M. L., Kremer, J., Bennett, A., Morsy, M., Eisen, J. A., Leach, J. E., and Dangl, J. L. 2017. Research priorities for harnessing plant microbiomes in sustainable agriculture. PLoS Biol. 15:e2001793.

Caporaso, J. G., Kuczynski, J., Stombaugh, J., Bittinger, K., Bushman, F. D., Costello, E. K., Fierer, N., Peña, A. G., Goodrich, J. K., Gordon, J. I., Huttley, G. A., Kelley, S. T., Knights, D., Koenig, J. E., Ley, R. E., Lozupone, C. A., McDonald, D., Muegge, B. D., Pirrung, M., Reeder, J., Sevinsky, J. R., Turnbaugh, P. J., Walters, W. A., Widmann, J., Yatsunenko, T., Zaneveld, J., and Knight, R. 2010. QIIME allows analysis of high-throughput community sequencing data. Nat. Methods 7:335-336.

Chen, Q., Hou, L. W., Duan, W. J., Crous, P. W., and Cai, L. 2017. Didymellaceae revisited. Stud. Mycol. 87:105-159.

Cherney, J. H., and Small, E. 2016. Industrial hemp in North America: Production, politics and potential. Agronomy (Basel) 6:58.

Colombo, C., Palumbo, G., He, J.-Z., Pinton, R., and Cesco, S. 2014. Review on iron availability in soil: Interaction of Fe minerals, plants, and microbes. J. Soils Sediments 14:538-548.

Comeau, D., Novinscak, A., Joly, D. L., and Filion, M. 2020. Spatio-temporal and cultivar-dependent variations in the cannabis microbiome. Front. Microbiol. 11:491.

Copeland, J. K., Yuan, L., Layeghifard, M., Wang, P. W., and Guttman, D. S. 2015. Seasonal community succession of the phyllosphere microbiome. Mol. Plant-Microbe Interact. 28:274-285.

de Cássia Silva, U., de Oliveira, C. A., de Paula Lana, U. G., Gomes, E. A., and dos Santos, V. L. 2016. Growth promotion features of the maize microbiome: From an agriculture perspective. Pages 345-374 in: Plant-Microbe Interaction: An Approach to Sustainable Agriculture. D. K. Choudhary, A. Varma, and N. Tuteja, eds. Springer Singapore, Singapore.

Del Frari, G., Cabral, A., Nascimento, T., Boavida Ferreira, R., and Oliveira, H. 2019. Epicoccum layuense a potential biological control agent of escaassociated fungi in grapevine. PLoS One 14:e0213273.

Delmotte, N., Knief, C., Chaffron, S., Innerebner, G., Roschitzki, B., Schlapbach, R., von Mering, C., and Vorholt, J. A. 2009. Community proteogenomics reveals insights into the physiology of phyllosphere bacteria. Proc. Natl. Acad. Sci. U.S.A. 106:16428-16433.

Di Cagno, R., Coda, R., De Angelis, M., and Gobbetti, M. 2013. Exploitation of vegetables and fruits through lactic acid fermentation. Food Microbiol. 33: $1-10$

Dubois, D., Pihet, M., Le Clec'h, C., Croué, A., Beguin, H., Bouchara, J. P., and Chabasse, D. 2005. Cutaneous phaeohyphomycosis due to Alternaria infectoria. Mycopathologia 160:117-123.

Edgar, R. C. 2010. Search and clustering orders of magnitude faster than BLAST. Bioinformatics 26:2460-2461.

Edwards, J., Johnson, C., Santos-Medellín, C., Lurie, E., Podishetty, N. K., Bhatnagar, S., Eisen, J. A., and Sundaresan, V. 2015. Structure, variation, and assembly of the root-associated microbiomes of rice. Proc. Natl. Acad. Sci. U.S.A. 112:E911-E920.

Franche, C., Lindström, K., and Elmerich, C. 2009. Nitrogen-fixing bacteria associated with leguminous and non-leguminous plants. Plant Soil 321:35-59.

Gautam, A. K., Kant, M., and Thakur, Y. 2013. Isolation of endophytic fungi from Cannabis sativa and study their antifungal potential. Arch. Phytopathol. Plant Prot. 46:627-635.

Golubev, W., Ikeda, R., Shinoda, T., and Nakase, T. 1997. Antifungal activity of Bullera alba (Hanna) Derx. Mycoscience 38:25-29.

Golzar, H., Thomas, G., Jayasena, K. W., Wright, D., Wang, C., and Kehoe, M. 2019. Neoascochyta species cause leaf scorch on wheat in Australia. Australas. Plant Dis. Notes 14:1.

Grady, K. L., Sorensen, J. W., Stopnisek, N., Guittar, J., and Shade, A. 2019. Assembly and seasonality of core phyllosphere microbiota on perennial biofuel crops. Nat. Commun. 10:4135.

Guo, L., Wang, G., Sheng, Y., Shi, Z., and Sun, X. 2019. Groundwater microbial communities and their connection to hydrochemical environment in Golmud, Northwest China. Sci. Total Environ. 695:133848.

Gyaneshwar, P., Naresh Kumar, G., Parekh, L. J., and Poole, P. S. 2002. Role of soil microorganisms in improving P nutrition of plants. Plant Soil 245:83-93.

Han, J.-I., Choi, H.-K., Lee, S.-W., Orwin, P. M., Kim, J., LaRoe, S. L., Kim, T., O’Neil, J., Leadbetter, J. R., Lee, S. Y., Hur, C., Spain, J. C., Ovchinnikova, G., Goodwin, L., and Han, C. 2011. Complete genome sequence of the metabolically versatile plant growth-promoting endophyte Variovorax paradoxus S110. J. Bacteriol. 193:1183-1190.

Hothorn, T., Bretz, F., and Westfall, P. 2008. Simultaneous inference in general parametric models. Biometrical J. 50:346-363.

Innerebner, G., Knief, C., and Vorholt, J. A. 2011. Protection of Arabidopsis thaliana against leaf-pathogenic Pseudomonas syringae by Sphingomonas strains in a controlled model system. Appl. Environ. Microbiol. 77: 3202-3210.

Ivanova, E. G., Doronina, N. V., Shepelyakovskaya, A. O., Laman, A. G., Brovko, F. A., and Trotsenko, Y. A. 2000. Facultative and obligate aerobic Methylobacteria synthesize cytokinins. Microbiology 69:646-651.

Ivanova, E. G., Doronina, N. V., and Trotsenko, Y. A. 2001. Aerobic Methylobacteria are capable of synthesizing auxins. Microbiology 70: 392-397.

Kalmbach, S., Manz, W., Bendinger, B., and Szewzyk, U. 2000. In situ probing reveals Aquabacterium commune as a widespread and highly abundant bacterial species in drinking water biofilms. Water Res. 34:575-581.

Kloepper, J. W., Ryu, C.-M., and Zhang, S. 2004. Induced systemic resistance and promotion of plant growth by Bacillus spp. Phytopathology 94 : 1259-1266.

Koechli, C., Campbell, A. N., Pepe-Ranney, C., and Buckley, D. H. 2019. Assessing fungal contributions to cellulose degradation in soil by using highthroughput stable isotope probing. Soil Biol. Biochem. 130:150-158.

Kozich, J. J., Westcott, S. L., Baxter, N. T., Highlander, S. K., and Schloss, P. D. 2013. Development of a dual-index sequencing strategy and curation pipeline for analyzing amplicon sequence data on the MiSeq Illumina sequencing platform. Appl. Environ. Microbiol. 79:5112-5120.

Kuklinsky-Sobral, J., Araújo, W. L., Mendes, R., Geraldi, I. O., PizziraniKleiner, A. A., and Azevedo, J. L. 2004. Isolation and characterization of soybean-associated bacteria and their potential for plant growth promotion. Environ. Microbiol. 6:1244-1251.

Kusari, P., Kusari, S., Spiteller, M., and Kayser, O. 2013. Endophytic fungi harbored in Cannabis sativa L.: Diversity and potential as biocontrol agents against host plant-specific phytopathogens. Fungal Divers. 60:137-151.

Law, D. A., McNees, R. C., and Moe, A. L. 2020. The microbiology of hemp retting in a controlled environment: Steering the hemp microbiome towards more consistent fiber production. Agronomy (Basel) 10:492.

Lievens, K. H., van Rijsbergen, R., Leyns, F. R., Lambert, B. J., Terming, P., Swings, J., and Joos, H. J.-P. 1989. Dominant rhizosphere bacteria as a source for antifungal agents. Pestic. Sci. 27:141-154.

Lopes, L., Borges-Costa, J., Soares-Almeida, L., Filipe, P., Neves, F., Santana, A., Guerra, J., and Kutzner, H. 2013. Cutaneous alternariosis caused by Alternaria infectoria: Three cases in kidney transplant patients. Health Care (Don Mills) 1:100-106.

Love, M. I., Huber, W., and Anders, S. 2014. Moderated estimation of fold change and dispersion for RNA-seq data with DESeq2. Genome Biol. 15:550.

McKernan, K., Spangler, J., Zhang, L., Tadigotla, V., Helbert, Y., Foss, T., and Smith, D. 2015. Cannabis microbiome sequencing reveals several mycotoxic fungi native to dispensary grade Cannabis flowers. F1000 Res. 4:1422.

McMurdie, P. J., and Holmes, S. 2013. phyloseq: An R package for reproducible interactive analysis and graphics of microbiome census data. PLoS One 8: e61217.

McPartland, J. M., and Cubeta, M. A. 1997. New species, combinations, host associations and location records of fungi associated with hemp (Cannabis sativa). Mycol. Res. 101:853-857.

Ofek, M., Hadar, Y., and Minz, D. 2012. Ecology of root colonizing Massilia (Oxalobacteraceae). PLoS One 7:e40117.

Ogle, D. H., Wheeler, P., and Dinno, A. 2020. FSA: Fisheries stock analysis. https:/github.com/droglenc/FSA

Oksanen, J., Blanchet, F. G., Friendly, M., Kindt, R., Legendre, P., McGlinn, D., Minchin, P. R., O’Hara, R. B., Simpson, G. L., Solymos, P., Stevens, M. H. H., Szoecs, E., and Wagner, H. 2018. vegan: Community Ecology Package. https://cran.r-project.org/web/packages/vegan/index.html

Ortíz-Castro, R., Contreras-Cornejo, H. A., Macías-Rodríguez, L., and LópezBucio, J. 2009. The role of microbial signals in plant growth and development. Plant Signal. Behav. 4:701-712.

Palmer, J. M., Jusino, M. A., Banik, M. T., and Lindner, D. L. 2018. Nonbiological synthetic spike-in controls and the AMPtk software pipeline improve mycobiome data. PeerJ 6:e4925.

Peiffer, J. A., Spor, A., Koren, O., Jin, Z., Tringe, S. G., Dangl, J. L., Buckler, E. S., and Ley, R. E. 2013. Diversity and heritability of the maize rhizosphere microbiome under field conditions. Proc. Natl. Acad. Sci. U.S.A. 110: 6548-6553. 
Perelló, A. E., and Sisterna, M. N. 2006. Leaf blight of wheat caused by Alternaria triticina in Argentina. Plant Pathol. 55:303.

Pieterse, C. M. J., Zamioudis, C., Berendsen, R. L., Weller, D. M., Van Wees, S. C. M., and Bakker, P. A. H. M. 2014. Induced systemic resistance by beneficial microbes. Annu. Rev. Phytopathol. 52:347-375.

Prasada, R., and Prabhu, A. S. 1962. Leaf blight of wheat caused by a new species of Alternaria. Indian Phytopathol. 15:292-293.

Rastogi, G., Coaker, G. L., and Leveau, J. H. J. 2013. New insights into the structure and function of phyllosphere microbiota through high-throughput molecular approaches. FEMS Microbiol. Lett. 348:1-10.

R Core Team. 2018. R: A Language and Environment for statistical Computing. The R Foundation, Vienna, Austria. https://www.r-project.org/

Rognes, T., Flouri, T., Nichols, B., Quince, C., and Mahé, F. 2016. VSEARCH: A versatile open source tool for metagenomics. PeerJ 4:e2584.

Saleem, M., Meckes, N., Pervaiz, Z. H., and Traw, M. B. 2017. Microbial interactions in the phyllosphere increase plant performance under herbivore biotic stress. Front. Microbiol. 8:41.

Schloss, P. D., Westcott, S. L., Ryabin, T., Hall, J. R., Hartmann, M., Hollister, E. B., Lesniewski, R. A., Oakley, B. B., Parks, D. H., Robinson, C. J., Sahl, J. W., Stres, B., Thallinger, G. G., Van Horn, D. J., and Weber, C. F. 2009. Introducing mothur: Open-source, platform-independent, communitysupported software for describing and comparing microbial communities. Appl. Environ. Microbiol. 75:7537-7541.

Schmalenberger, A., Hodge, S., Bryant, A., Hawkesford, M. J., Singh, B. K., and Kertesz, M. A. 2008. The role of Variovorax and other Comamonadaceae in sulfur transformations by microbial wheat rhizosphere communities exposed to different sulfur fertilization regimes. Environ. Microbiol. 10:1486-1500.

Schmalenberger, A., and Tebbe, C. C. 2003. Bacterial diversity in maize rhizospheres: Conclusions on the use of genetic profiles based on PCRamplified partial small subunit rRNA genes in ecological studies. Mol. Ecol. 12:251-262.

Schmidt, J. E., Kent, A. D., Brisson, V. L., and Gaudin, A. C. M. 2019. Agricultural management and plant selection interactively affect rhizosphere microbial community structure and nitrogen cycling. Microbiome 7:146.

Scott, M., Rani, M., Samsatly, J., Charron, J. B., and Jabaji, S. 2018. Endophytes of industrial hemp (Cannabis sativa L.) cultivars: Identification of culturable bacteria and fungi in leaves, petioles, and seeds. Can. J. Microbiol. 64: 664-680.

Small, E., and Cronquist, A. 1976. A practical and natural taxonomy for Cannabis. Taxon 25:405-435.

Taghinasab, M., and Jabaji, S. 2020. Cannabis microbiome and the role of endophytes in modulating the production of secondary metabolites: An overview. Microorganisms 8:355.

Toju, H., Peay, K. G., Yamamichi, M., Narisawa, K., Hiruma, K., Naito, K., Fukuda, S., Ushio, M., Nakaoka, S., Onoda, Y., Yoshida, K., Schlaeppi, K., Bai, Y., Sugiura, R., Ichihashi, Y., Minamisawa, K., and Kiers, E. T. 2018. Core microbiomes for sustainable agroecosystems. Nat. Plants 4:247-257.
Trotsenko, Y. A., Ivanova, E. G., and Doronina, N. V. 2001. Aerobic methylotrophic bacteria as phytosymbionts. Microbiology 70:623-632.

Turner, T. R., James, E. K., and Poole, P. S. 2013. The plant microbiome. Genome Biol. 14:209.

Urquhart, E. J., Menzies, J. G., and Punja, Z. K. 1994. Growth and biological control activity of Tilletiopsis species against powdery mildew (Sphaerotheca fuliginea) on greenhouse cucumber. Phytopathology 84:341-351.

Urquhart, E. J., and Punja, Z. K. 2002. Hydrolytic enzymes and antifungal compounds produced by Tilletiopsis species, phyllosphere yeasts that are antagonists of powdery mildew fungi. Can. J. Microbiol. 48:219-229.

Vorholt, J. A. 2012. Microbial life in the phyllosphere. Nat. Rev. Microbiol. 10: 828-840.

Wallace, J. G., Kremling, K. A., Kovar, L. L., and Buckler, E. S. 2018. Quantitative genetics of the maize leaf microbiome. Phytobiomes J. 2:208-224.

Walters, W. A., Jin, Z., Youngblut, N., Wallace, J. G., Sutter, J., Zhang, W., González-Peña, A., Peiffer, J., Koren, O., Shi, Q., Knight, R., Glavina del Rio, T., Tringe, S. G., Buckler, E. S., Dangl, J. L., and Ley, R. E. 2018. Large-scale replicated field study of maize rhizosphere identifies heritable microbes. Proc. Natl. Acad. Sci. U.S.A. 115:7368-7373.

Wang, H., Wang, S. D., Jiang, Y., Zhao, S. J., and Chen, W. X. 2014. Diversity of rhizosphere bacteria associated with different soybean cultivars in two soil conditions. Soil Sci. Plant Nutr. 60:630-639.

Wei, Z., Huang, J., Tan, S., Mei, X., Shen, Q., and Xu, Y. 2013. The congeneric strain Ralstonia pickettii QL-A6 of Ralstonia solanacearum as an effective biocontrol agent for bacterial wilt of tomato. Biol. Control 65:278-285.

Weiss, S., Xu, Z. Z., Peddada, S., Amir, A., Bittinger, K., Gonzalez, A., Lozupone, C., Zaneveld, J. R., Vázquez-Baeza, Y., Birmingham, A., Hyde, E. R., and Knight, R. 2017. Normalization and microbial differential abundance strategies depend upon data characteristics. Microbiome 5:27.

Weldon, W. A., Ullrich, M. R., Smart, L. B., Smart, C. D., and Gadoury, D. M. 2020. Cross-infectivity of powdery mildew isolates originating from hemp (Cannabis sativa) and Japanese hop (Humulus japonicus) in New York. Plant Health Prog. 21:47-53.

Weller, D. M. 1988. Biological control of soilborne plant pathogens in the rhizosphere with bacteria. Annu. Rev. Phytopathol. 26:379-407.

Wellner, S., Lodders, N., and Kämpfer, P. 2011. Diversity and biogeography of selected phyllosphere bacteria with special emphasis on Methylobacterium spp. Syst. Appl. Microbiol. 34:621-630.

Whiting, S. N., de Souza, M. P., and Terry, N. 2001. Rhizosphere bacteria mobilize $\mathrm{Zn}$ for hyperaccumulation by Thlaspi caerulescens. Environ. Sci. Technol. 35:3144-3150.

Winston, M. E., Hampton-Marcell, J., Zarraonaindia, I., Owens, S. M., Moreau, C. S., Gilbert, J. A., Hartsel, J., Kennedy, S. J., and Gibbons, S. M. 2014. Understanding cultivar-specificity and soil determinants of the Cannabis microbiome. PLoS One 9:e99641.

Zhang, J., Kobert, K., Flouri, T., and Stamatakis, A. 2014. PEAR: A fast and accurate Illumina Paired-End reAd mergeR. Bioinformatics 30:614-620. 